\title{
High-Efficiency Light-Emitting Diodes Based on Formamidinium Lead Bromide Nanocrystals and solution processed transport layers
}

\author{
Francesco Di Stasio ${ }^{\dagger}$, Iñigo Ramiro ${ }^{+, \neq}, \mathrm{Yu} \mathrm{Bi}^{+, \neq}$, Sotirios $\mathrm{Christodoulou}^{+}$, Alexandros Stavrinadis $^{\dagger}$ and Gerasimos \\ Konstantatos ${ }^{\dagger, *}$ \\ +ICFO-Institut de Ciencies Fotoniques, The Barcelona Institute of Science and Technology, 08860 Castelldefels (Barcelona), Spain \\ *ICREA-Institució Catalana de Recerca i Estudis Avançats, Passeig Lluís Companys 23, 08010 Barcelona, Spain \\ KEYWORDS light-emitting diodes, perovskite, formamidinium, nanocrystals, quantum-dots
}

\begin{abstract}
Perovskite nanocrystal light-emitting diodes (LEDs) employing architecture comprising a ZnO nanoparticles electrontransport layer and a conjugated polymer hole-transport layer have been fabricated. The obtained LEDs demonstrate a maximum externalquantum-efficiency of $6.04 \%$, luminance of $12998 \mathrm{Cd} / \mathrm{m}^{2}$ and stable electroluminescence at $519 \mathrm{~nm}$. Importantly, such high efficiency and brightness have been achieved by employing solution processed transport layers, formamidinium lead bromide nanocrystals $\left(\mathrm{CH}\left(\mathrm{NH}_{2}\right)_{2} \mathrm{PbBr}_{3} \mathrm{NCs}\right)$ synthesized at room-temperature and in air without the use of a Schlenk line, and a procedure based on atomic layer deposition to insolubilize the NC film. The obtained NCs show a photoluminescence quantum yield of $90 \%$ that is retained upon film fabrication. The results show that perovskite NC LEDs can achieve high-performance without the use of transport layers deposited through evaporation in ultra-high-vacuum.
\end{abstract}

Perovskite nanocrystals (NCs) ${ }^{1}$ have been successfully applied in a variety of optoelectronic devices such as solar cells, ${ }^{2-}$ ${ }^{4}$ solar concentrators, ${ }^{5}$ lasers, ${ }^{67}$ white phosphors ${ }^{8}$ and light-emitting diodes (LEDs) ${ }^{9-13}$ Since the synthesis of CsPbX ${ }_{3} \mathrm{NCs}$ (where $\mathrm{X}=\mathrm{Cl}, \mathrm{Br}$ or I) was demonstrated in $2015,{ }^{14}$ attention toward this class of perovskite materials has considerably grown, and more effort has been dedicated in exploiting their favourable optical properties. In particular, focus has been placed on the fabrication of efficient light-emitting diodes (LEDs), since perovskite NCs possess desirable light-emission properties such as tuneable colour and narrow emission, which could lead to colour-pure devices for application in displays and illumination. Another important aspect is that perovskite NCs possess a defect-tolerant structure ${ }^{1}$ where defect states do not have a strong influence on the radiative recombination, thus leading to high photoluminescence quantum yield (PLQY) without the need of surface passivation. Nevertheless, major challenges are still present as the labile nature of the surface ligands combined with the strong ionic character of the structure are causes of instability; ${ }^{15}$ and purification of the NCs after synthesis requires particular attention as use of anti-solvents can irreversibly damage the material. ${ }^{4}$ As a result, their surface chemistry has been studied and tailored ${ }^{4,7,15}$ to increase their stability, while purification procedures have been developed to avoid degradation. ${ }^{4,10}$ These developments have allowed the fabrication of efficient LEDs and currently the best perovskite NC LEDs show a maximum external-quantum-efficiency (EQE) of $12.9 \%{ }^{16}$ Such performance has been achieved in a device structure employing an electron-transport layer prepared trough thermal-evaporation in ultra-high-vacuum conditions. Yet, device architectures based on solution-processed materials, which do not require lengthy and expensive fabrication procedures, are still trailing behind with EQEs in the region of only few percentages. ${ }^{11,17}$ The discrepancy in performance has a twofold explanation: first, perovskite NCs possess limited thermal stability which impedes annealing (typically employed to partially insolubilize the layer) without damaging the film. ${ }^{18}$ Secondly, antisolvents (i.e. polar solvents) cannot be used during the spin-coating of top layers as the perovskite NC film will be damage as previously discussed. ${ }^{4}$

Here, we report an approach to overcome these challenges and obtain highly efficient fully-solution processed LEDs based on $\mathrm{CH}\left(\mathrm{NH}_{2}\right)_{2} \mathrm{PbBr}_{3} \mathrm{NCs}\left(\mathrm{FAPbBr}_{3}\right)$. Substituting Cs with formamidinium (FA) in our synthesis, we obtained NCs which are more resistant to the device fabrication procedure. The obtained $\mathrm{FAPbBr}_{3} \mathrm{NC}$ film was insolubilized using a procedure based on atomic layer deposition (ALD). The obtained devices show a maximum EQE of 6.04\% and a maximum luminance of $12998 \mathrm{Cd} / \mathrm{m}^{2}$.

Figure 1a presents a scheme and focused-ion-beam cross-section of the fabricated devices (see Figure S1 in the supporting information for the ultra-violet photoelectron spectroscopy spectra used to derive the $\mathrm{FAPbBr}_{3} \mathrm{NC}_{\text {film valence band }}$ 
position with respect to vacuum). As electron-transport layer we have employed a $\mathrm{ZnO}$ nanoparticles ${ }^{19}$ film deposited via spin-coating on a patterned-ITO/glass substrate, while for hole injection/transport we have used a bi-layer structure comprising poly vinyl carbazole (PVK) and Poly[N,N'-bis(4-butylphenyl)-N,N'-bisphenylbenzidine] (PTPD). Such a holetransport layer has been previously successfully employed in LEDs based on cadmium chalcogenides NCs as it combines the high hole mobility from the PTPD and the enhanced electron blocking capabilities from the PVK. ${ }^{20,21}$ The device was completed via sputtering deposition of a Pt electrode, and encapsulation with epoxy glue and a glass slide. The energy diagram in Figure $1 \mathrm{~b}$ shows that only small energy barriers are present at the active-layer/transport-layer interface in the LEDs, namely $0.4 \mathrm{eV}$ for electrons and $0.5 \mathrm{eV}$ for holes.

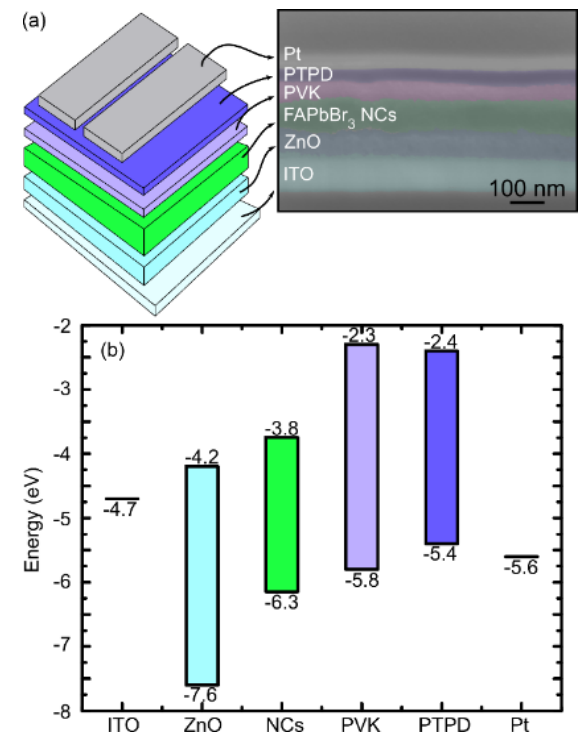

Figure 1. (a) Scheme of the fully solution-processed structure used in the light-emitting diodes and FIB SEM cross-sectional image of one device. (b) Energy level diagram of the materials that constitute the LED. The energy values for the various device components were taken from literature, while the values reported for the $\mathrm{FAPbBr} 3 \mathrm{NCs}$ were obtained from ultra-violet photoelectron spectroscopy of a film deposited on ITO, and its optical absorption spectrum.

In Figure 2a we report the current density-luminance-voltage (JVL) curve for the "champion device" (i.e. LED with highest EQE). The LED reaches a maximum luminance of $12998 \mathrm{~cd} / \mathrm{m}^{2}$; a remarkable value but lower than the 22830 $\mathrm{cd} / \mathrm{m}^{2}$ achieved in the highest EQE perovskite LED based on evaporated transport layers, ${ }^{16}$ while LEDs based on bulk $\mathrm{CsPbBr}{ }_{3}$ can achieve higher luminance compared to NCs. ${ }^{22}$ From the JVL curves we can estimate a turn-on voltage $\left(\mathrm{V}_{\text {on }}\right.$, extracted from a luminance of $0.1 \mathrm{~cd} / \mathrm{m}^{2}$, see Figure S2) of $4.1 \mathrm{~V}$, which is higher than the estimated optical band-gap of the $\mathrm{FAPbBr}_{3} \mathrm{NCs}(2.43 \mathrm{eV})$. In fact, in an ideal LED, the lowest limit of the $\mathrm{V}_{\text {on }}$ is represented by the optical band gap of the emitting material; ${ }^{23}$ yet many factors during device fabrication can induce increased $\mathrm{V}_{\text {on. }}$. In our case, we assign the relatively high $\mathrm{V}_{\text {on }}$ to the increased thickness of the LED and a corresponding increase in series resistance. We found that using a thick $\mathrm{FAPbBr}_{3} \mathrm{NC}$ layer $(95 \mathrm{~nm})$ allows the device to reach higher luminance and higher EQE at the cost of an increased operational voltage (see Table S1 in the supporting information). The cross-section of the LED in Figure 1a reveals that the overall device thickness is $\approx 270 \mathrm{~nm}$, thus corroborating our explanation. Despite the high operating voltage, the champion device shows a maximum EQE of $6.04 \%$ (Figure $2 \mathrm{~b}$ ) and a maximum current efficiency of $20.53 \mathrm{~cd} / \mathrm{A}$. To our knowledge, these are the highest efficiency values reported for perovskite NC LEDs based on solution processed transport layers to date, and the champion device outperforms other LEDs based on $\mathrm{FAPbBr}_{3} \mathrm{NCs}$ as well. No drastic efficiency droop is observed for high driving currents: the EQE is reduced only by $10 \%$ from 20 to $68.8 \mathrm{~mA} / \mathrm{cm}^{2}$ (i.e., 6.3 to $8.4 \mathrm{~V}$ ).). The efficiency droop in NC LED is known to be caused by Auger recombination at high driving current density when charge balance in the active layer is not achieved. The stable efficiency observed in our LEDs indicates that Auger recombination does not play a major role (i.e. in the active layer charge imbalances are reduced) allowing high luminance to be reached and the efficiency maintained. From the EQE value we can estimate the internal quantum efficiency (IQE) following a previously reported method, ${ }^{24}$ thus obtaining a maximum IQE of $25.54 \%$. The estimated IQE is lower than the measured PLQY for the $\mathrm{FAPbBr}_{3} \mathrm{NC}$ film used in the device of $46 \pm 5 \%$, indicating that further device optimization can increase the efficiency of these LEDs. The electroluminescence (EL) spectrum is stable under increasing applied bias (see Figure 2c, colour coordinates diagram is reported in Figure S3) up to 8.5V. The EL is centred at $519 \mathrm{~nm}$ with a full-width- 
half-maximum (FWHM) of $18 \mathrm{~nm}$, in the range of previously reported perovskite NC LEDs. ${ }^{10,16}$ The "champion device" here presented shows good performance in terms of EQE and maximum luminance and this result is reproducible in large part of our LEDs, as shown in the histogram in Figure 2d, where an average EQE of 5.16\% is calculated from a total of 30 different devices. Nevertheless, device stability remains very limited as the LEDs are operational for only few minutes, similar to what recently reported by F. Yan et al. ${ }^{16}$ The short operational lifetime is currently the main issue of perovskite NC devices and in our case we can observe a decrease in performance already after the initial JVL scan (see Figure S4).
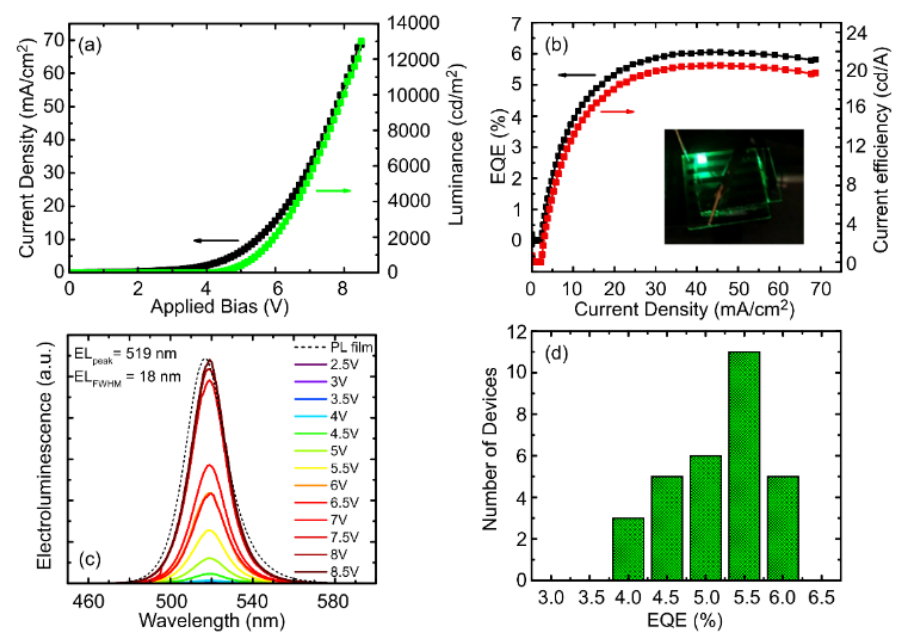

Figure 2. (a) Current density-Voltage-Luminance curves for the LED with highest EQE (champion device). (b) EQE and current efficiency curves vs current density, inset: photo of an LED under operation. (c) Electroluminescence spectra recorded at increasing applied voltage. For comparison the PL spectrum of the film is plotted (black dashed line). (d) Histogram of the achieved EQE for the LEDs.

We now focus our discussion on how we achieved such LED performance, starting from the material development and properties, to the fabrication of the active layer.

The $\mathrm{FAPbBr}_{3} \mathrm{NCs}$ were synthesized following our previously published procedure for $\mathrm{CsPbBr}_{3} \mathrm{NCs}^{25}$ where, instead of Cesium acetate, we have employed Formamidine acetate as a precursor (see experimental section and Table S2 in the supporting information for the details on the volumes and precursors used). The main motivations to substitute Cs with FA is the necessity to purify the obtained material to produce films with reduced roughness. Nevertheless, LEDs based on FA-containing perovskites demonstrate comparable performance to Cs-containing ones, both when synthesized as NCs or nanostructured film. ${ }^{26-28}$ In order to fabricate continuous solid films to be used in LEDs, we have developed a purification procedure that aims at reducing NC aggregation and removal of ligand excess without compromising the PLQY of the NCs. A schematic of the purification procedure employed is presented in Figure 3a. Importantly, $\mathrm{FAPbBr}_{3} \mathrm{NCs}$ demonstrate higher stability during purification compared to $\mathrm{CsPbr}_{3}$ synthesized with our procedure. In fact, in the case of CsP$\mathrm{bBr}_{3} \mathrm{NCs}$, high PLQY is readily observed directly after synthesis ${ }^{2,25}$ but further purification damages the NCs, thus showing a PLQY drop to $45 \pm 5 \%$. The obtained NC solution is initially centrifuged in order to transfer the NCs from the synthesis solvents mixture to anhydrous toluene. A post-synthesis treatment employing $\mathrm{PbBr}_{2}$ complexes (see reference 25) is then applied to the NCs to enhance further the PLQY: pristine NCs show a PLQY of 35\% directly after synthesis, the treatment enhances it up to $48 \%$. After the treatment with $\mathrm{PbBr}_{2}$, oleic acid is added in order to dissolve the NC aggregates that are observed in solution. Room-temperature synthetic approaches based on the use of relatively short-ligands are known to give rise to aggregation or coalescence during the NC crystallization. ${ }^{2,25}$ Presence of aggregates/coalescence can be observed in TEM images of the as-synthesized NCs (see Figure S5), and it is further indicated by the turbid colour of the solution (Figure S6). Employing as-synthesized NCs for film preparation does not allow for the fabrication of working LEDs due to the high roughness of the obtained films (RMS roughness $=20.95 \mathrm{~nm}$ ). Addition of few $\mu \mathrm{l}$ of oleic acid partially dissolves the aggregates (Figure $3 \mathrm{~b}$ and photo in Figure S6). After this step the NC were centrifuged at slow speed to remove the large not-dissolved aggregates, and finally they were washed one last time with methyl acetate to remove excess ligands in solution. ${ }^{4}$ Following this procedure we were able to fabricate spin-coated $\mathrm{FAPbBr}_{3} \mathrm{NC}$ films with an RMS roughness $=3.45 \mathrm{~nm}$. Figure $3 \mathrm{c}$ shows the optical absorption and $\mathrm{PL}$ spectra of the $\mathrm{FAPbBr}_{3} \mathrm{NCs}$ in toluene solution. The NCs present a PL peak at $511 \mathrm{~nm}$ and FWHM $=22 \mathrm{~nm}$. Spectral shape and PL peak position are unchanged compared to 
the not purified material. After the purification, the PLQY shows a 1.85 times increase up to $90 \pm 9 \%$. This enhancement is accompanied by a drastic change in the PL dynamics (Figure 3d) where the initial fast decay component reduces, thus increasing the PL lifetime from $2.66 \mathrm{~ns}$ to $4.27 \mathrm{~ns}$ (a summary of the PL decay fitting values is reported in Table S3). The PL lifetime after purification is 1.6 times longer than for the as-synthesized NCs. This increase is close to the one observed for the PLQY, thus indicating that the enhanced luminescence is correlated to a decrease in the non-radiative rate of the emitter (i.e. decrease of the fast component observed in the PL decay of the as-synthesized NCs). Films fabricated via spin-coating show a small red-shift of the PL peak (from $511 \mathrm{~nm}$ in solution to $516 \mathrm{~nm}$ in film), yet the PLQY is preserved, showing once again a value of $90 \pm 9 \%$. We have previously observed this surprisingly high PLQY in $\mathrm{CsPbBr}_{3} \mathrm{NCs}$ prepared with the very same synthetic method ${ }^{3,25}$ and it can be tentatively assigned to hole trap passivation carried out by ambient oxygen in the spin-coated film. ${ }^{29,30}$ These results show that the removal of aggregates in $\mathrm{FAPbBr}_{3} \mathrm{NCs}$ synthesized at room temperature and with short ligands is necessary to obtain highly luminescent material, in contrast with what observed for $\mathrm{Cs} \mathrm{PbBr}_{3} \mathrm{NCs}$.

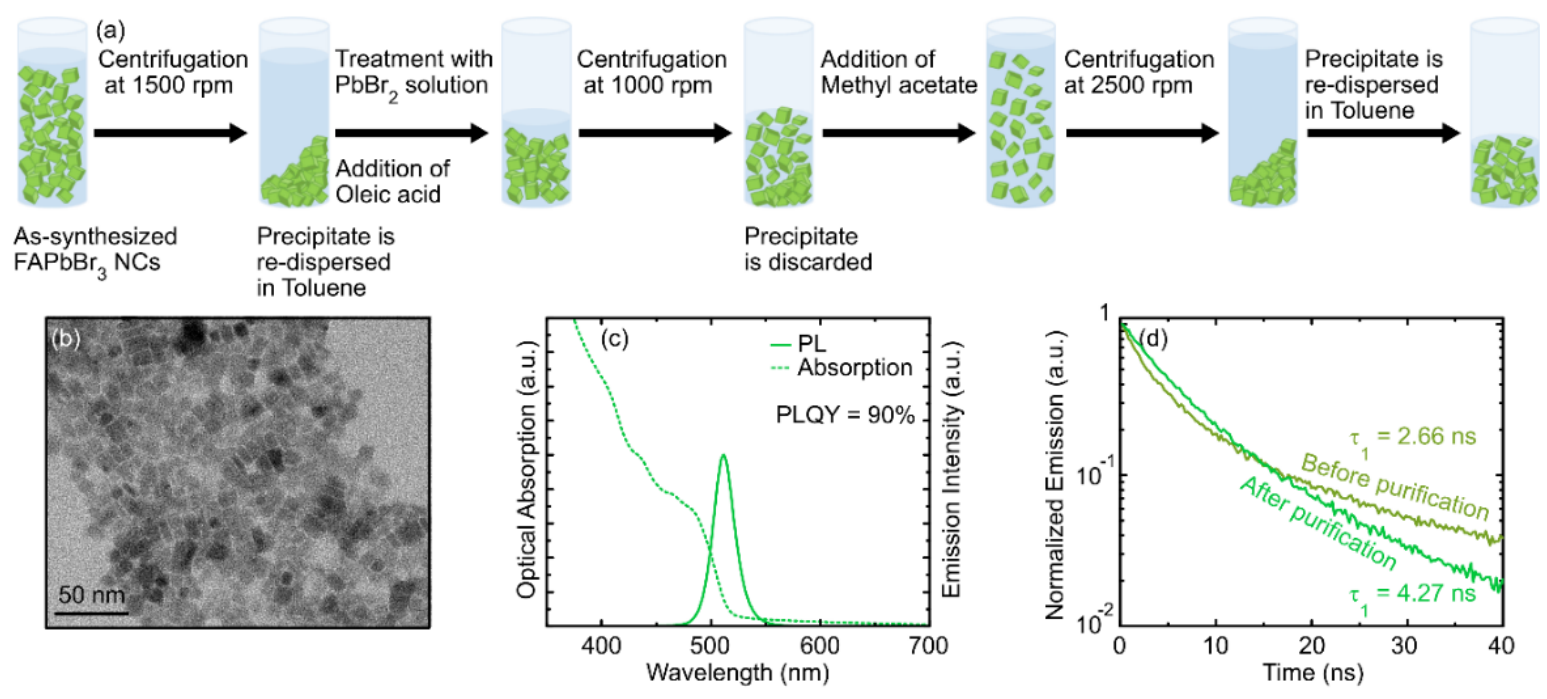

Figure 3. (a) Scheme of the purification procedure employed. The obtained $\mathrm{FAPbBr}_{3} \mathrm{NCs}$ were directly used for film fabrication via spin-coating after purification. (b) Transmission electron microscope micrograph of the $\mathrm{FAPbBr}_{3} \mathrm{NCs}$ after purification. (c) Optical absorption and steady-state PL spectrum of the purified $\mathrm{FAPbBr}_{3} \mathrm{NCs}$ in toluene solution. (c) Time-resolved PL of the $\mathrm{FAPbBr}_{3} \mathrm{NCs}_{3}$ in toluene solution before (dark green) and after (light green) purification, and magnification of the initial part of the PL decay (inset). The PL decays were fitted with a bi-exponential function and in the figure the dominant component $\left(\tau_{1}\right)$ is reported.

Following the substitution of Cs with FA in our NCs, we have employed a cross-linking method to prevent dissolution of the NC film upon spin-coating of the hole-transport layer, thus removing the need to carry out an annealing step in order to insolubilize the NC layer. As reported by G. Li et al., exposure of a NC film to trimethylaluminum (TMA) vapours in an ALD system, followed by storage in air, it induces partial cross-linking of the surface ligands, enabling high filmretention rates (i.e. thickness reduction upon spin-rinsing with a solvent). We have used a similar procedure for our FAP$\mathrm{bBr}_{3} \mathrm{NC}$ film. Yet, due to the different synthetic approach and the relatively short length of the surface ligands in our synthesis, we obtained substantially different results compared to what observed for perovskite NCs prepared via hotinjection method. This cross-linking method is advantageous compared to ultra-high vacuum deposition of transport layers as it requires only few minutes to be completed and it is carried out at room temperature. In Figure S7 we report the impact of the ALD treatment (see experimental section and supporting information) on the luminescence of the $\mathrm{FAPbBr}_{3}$ NC film spin-coated on glass. Directly after exposure of the film to TMA vapours, the PLQY of the film shows a drop from the original value of $90 \%$ to $<1 \%$ when 20 pulses of TMA vapours are applied. On the other hand, storage of the film in air at room-temperature for $24 \mathrm{~h}$ induces a $201 \%$ increase of the PLQY. This effect is somewhat similar to what previously reported ${ }^{9}$ but slower in comparison, as it is requires $24 \mathrm{~h}$ of exposure to air. This can be caused by the shorter ligands used in our synthesis (octylamine/octanoic acid) which slows down the kinetic of the cross-linking reaction. Importantly, we do not observe an enhancement of the PLQY but only a recovery during storage in air. Following these results, we have then assessed the film-retention of the cross-linked $\mathrm{FAPbBr}_{3} \mathrm{NC}$ film. We observed that already 5 pulses of TMA vapours 
were sufficient to decrease the thickness loss to $10 \%$, thus indicating that it is not necessary to sacrifice a substantial amount of luminescence to obtain an insoluble NC film. From these different parameters, we have found a trade-off condition between PLQY loss and film retention by using 3 pulses of TMA vapours, thus obtaining a film with a final PLQY of $46 \%$ and a film retention rate of $85 \%$. Considering the performance of our LEDs, this indicates that a retention rate of $100 \%$ is not required to achieve high EQE and Luminance, and certain degree of NC film/hole-transport layer intermixing can be accepted. SEM imaging carried out on $\mathrm{FAPbBr}_{3} \mathrm{NC}$ film before and after cross-linking reveal no sizeable changes in film morphology and no cracks are formed following the procedure (see Figure S8 in the supporting information).

In conclusion, we have fabricated highly efficient LEDs employing solution-processed materials combined with a crosslinking procedure based on ALD, thus demonstrating that is possible to achieve high performance without the use of evaporated transport layers. The obtained devices outperform current perovskite NC LEDs based on solution-processed transport layers (EQE 6.04\% vs 1.1\%) ${ }^{11}$ and LEDs based on $\mathrm{FAPbBr}_{3} \mathrm{NCs}(20.53 \mathrm{~cd} / \mathrm{A}$ vs $13.02 \mathrm{~cd} / \mathrm{A}) .{ }^{31}$ Importantly, solution processed LEDs based on quantum dots of different composition or shape, ${ }^{21,32}$ and conjugated polymers ${ }^{33}$ are now demonstrating very competitive performance. We hope that our work will encourage further developments for perovskite NCs towards all-solution processed LEDs.

\section{AUTHOR INFORMATION}

\section{Corresponding Author}

Gerasimos Konstantatos: Gerasimos.Konstantatos@icfo.eu

Francesco Di Stasio: Francesco.Distasio@icfo.eu

\section{Author Contributions}

$\$ \neq I$. R. and Y. B. contributed equally to this work

\section{Funding Sources}

The authors acknowledge financial support from the European Research Council (ERC) under the European Union's Horizon 2020 research and innovation programme (grant agreement no. 725165), the Spanish Ministry of Economy and Competitiveness (MINECO), and the "Fondo Europeo de Desarrollo Regional" (FEDER) through grant TEC201788655-R and the program redes de excellencia TFE by MINECO. The authors also acknowledge financial support from Fundacio Privada Cellex, the program CERCA and from the Spanish Ministry of Economy and Competitiveness, through the "Severo Ochoa" Programme for Centres of Excellence in R\&D (SEV-2015-0522). F. Di Stasio and S. Christodoulou acknowledge support from two Marie Curie Standard European Fellowships ("NANOPTO”, H2020-MSCA-IF-2015703018 and "NAROBAND", H2020-MSCA-IF-2016-750600). I. Ramiro acknowledges support from the Ministerio de Economía, Industria y Competitividad of Spain via a Juan de la Cierva fellowship.

\section{REFERENCES}

(1) Akkerman, Q. A.; Rainò, G.; Kovalenko, M. V; Manna, L. Genesis, Challenges and Opportunities for Colloidal Lead Halide Perovskite Nanocrystals. Nat. Mater. 2018, 17, 394-405.

(2) Akkerman, Q. A.; Gandini, M.; Di Stasio, F.; Rastogi, P.; Palazon, F.; Bertoni, G.; Ball, J. M.; Prato, M.; Petrozza, A.; Manna, L. Strongly Emissive Perovskite Nanocrystal Inks for High-Voltage Solar Cells. Nat. Energy 2017, 2 , 16194.

(3) Christodoulou, S.; Di Stasio, F.; Pradhan, S.; Stavrinadis, A.; Konstantatos, G. High-Open-Circuit-Voltage Solar Cells Based on Bright Mixed-Halide CsPbBrI $<$ inf $>2</$ Inf $>$ Perovskite Nanocrystals Synthesized under Ambient Air Conditions. J. Phys. Chem. C2018, 122, 7621-7626.

(4) Swarnkar, A.; Marshall, A. R.; Sanehira, E. M.; Chernomordik, B. D.; Moore, D. T.; Christians, J. A.; Chakrabarti, T.; Luther, J. M. Quantum Dot-induced Phase Stabilization of $\alpha-\mathrm{CsPbI}_{3}$ Perovskite for High-Efficiency Photovoltaics. Science 2016, 354, 92-95..

(5) Meinardi, F.; Akkerman, Q. A.; Bruni, F.; Park, S.; Mauri, M.; Dang, Z.; Manna, L.; Brovelli, S. Doped Halide Perovskite Nanocrystals for Reabsorption-Free Luminescent Solar Concentrators. ACS Energy Lett. 2017, 2, 2368-2377.

(6) Nedelcu, G.; Protesescu, L.; Yakunin, S.; Bodnarchuk, M. I.; Grotevent, M. J.; Kovalenko, M. V. Fast AnionExchange in Highly Luminescent Nanocrystals of Cesium Lead Halide Perovskites (CsPbX3, X = Cl, Br, I). Nano 
Lett. 2015, 15, 5635-5640.

(7) Pan, J.; Sarmah, S. P.; Murali, B.; Dursun, I.; Peng, W.; Parida, M. R.; Liu, J.; Sinatra, L.; Alyami, N.; Zhao, C.; Alarousu, E.; Ng, T. K.; Ooi, B. S.; Bakr, O. M.; Mohammed, O. F. Air-Stable Surface-Passivated Perovskite Quantum Dots for Ultra-Robust, Single- and Two-Photon-Induced Amplified Spontaneous Emission. J. Phys. Chem. Lett. 2015, 6, 5027-5033.

(8) Palazon, F.; Di Stasio, F.; Akkerman, Q. A.; Krahne, R.; Prato, M.; Manna, L. Polymer-Free Films of Inorganic Halide Perovskite Nanocrystals as UV-to-White Color-Conversion Layers in LEDs. Chem. Mater. 2016, 28, 29022906.

(9) Li, G.; Rivarola, F. W. R.; Davis, N. J. L. K.; Bai, S.; Jellicoe, T. C.; de la Peña, F.; Hou, S.; Ducati, C.; Gao, F.; Friend, R. H.; Greenham, N. C.; Tan, Z.-K. Highly Efficient Perovskite Nanocrystal Light-Emitting Diodes Enabled by a Universal Crosslinking Method. Adv. Mater. 2016, 28, 3528-3534.

(10) Chiba, T.; Hoshi, K.; Pu, Y.-J.; Takeda, Y.; Hayashi, Y.; Ohisa, S.; Kawata, S.; Kido, J. High-Efficiency Perovskite Quantum-Dot Light-Emitting Devices by Effective Washing Process and Interfacial Energy Level Alignment. ACS Appl. Mater. Interfaces 2017, 9, 18054-18060.

(11) Shamsi, J.; Rastogi, P.; Caligiuri, V.; Abdelhady, A. L.; Spirito, D.; Manna, L.; Krahne, R. Bright-Emitting Perovskite Films by Large-Scale Synthesis and Photoinduced Solid-State Transformation of CsPbBr3 Nanoplatelets. ACS Nano 2017, 11, 10206-10213.

(12) Veldhuis, S. A.; Ng, Y. F.; Ahmad, R.; Bruno, A.; Jamaludin, N. F.; Damodaran, B.; Mathews, N.; Mhaisalkar, S. G. Crown Ethers Enable Room-Temperature Synthesis of CsPbBr3 Quantum Dots for Light-Emitting Diodes. ACS Energy Lett. 2018, 3, 526-531.

(13) Kim, Y.; Yassitepe, E.; Voznyy, O.; Comin, R.; Walters, G.; Gong, X.; Kanjanaboos, P.; Nogueira, A. F.; Sargent, E. H. Efficient Luminescence from Perovskite Quantum Dot Solids. ACS Appl. Mater. Interfaces 2015, 7, 2500725013.

(14) Protesescu, L.; Yakunin, S.; Bodnarchuk, M. I.; Krieg, F.; Caputo, R.; Hendon, C. H.; Yang, R. X.; Walsh, A.; Kovalenko, M. V. Nanocrystals of Cesium Lead Halide Perovskites (CsPbX3, X $=\mathrm{Cl}, \mathrm{Br}$, and I): Novel Optoelectronic Materials Showing Bright Emission with Wide Color Gamut. Nano Lett. 2015, 15, 3692-3696.

(15) De Roo, J.; Ibáñez, M.; Geiregat, P.; Nedelcu, G.; Walravens, W.; Maes, J.; Martins, J. C.; Van Driessche, I.; Kovalenko, M. V; Hens, Z. Highly Dynamic Ligand Binding and Light Absorption Coefficient of Cesium Lead Bromide Perovskite Nanocrystals. ACS Nano 2016, 10, 2071-2081.

(16) Yan, F.; Xing, J.; Xing, G.; Quan, L.; Tan, S. T.; Zhao, J.; Su, R.; Zhang, L.; Chen, S.; Zhao, Y.; Huan, A.; Sargent, E. H.; Xiong, Q.; Demir, H. V. Highly Efficient Visible Colloidal Lead-Halide Perovskite Nanocrystal Light-Emitting Diodes. Nano Lett. 2018, 18, 3157-3164.

(17) Hung-Chia, W.; Zhen, B.; Hsin-Yu, T.; An-Cih, T.; Ru-Shi, L. Perovskite Quantum Dots and Their Application in Light-Emitting Diodes. Small 2017, 14, 1702433.

(18) Palazon, F.; Di Stasio, F.; Lauciello, S.; Krahne, R.; Prato, M.; Manna, L. Evolution of $\mathrm{CsPbBr}<\inf >3</$ Inf $>$ nanocrystals upon Post-Synthesis Annealing under an Inert Atmosphere. J. Mater. Chem. C 2016, 4, 9179-9182.

(19) K., R. A.; F., P. G. de A.; Alexandros, S.; Tania, L.; Maria, B.; L., D. S.; Gerasimos, K. Remote Trap Passivation in Colloidal Quantum Dot Bulk Nano-heterojunctions and Its Effect in Solution-Processed Solar Cells. Adv. Mater. 2014, 26, 4741-4747.

(20) Rastogi, P.; Palazon, F.; Prato, M.; Di Stasio, F.; Krahne, R. Enhancing the Performance of CdSe/CdS Dot-in-Rod Light-Emitting Diodes via Surface Ligand Modification. ACS Appl. Mater. Interfaces 2018, 10, 5665-5672.

(21) Dai, X.; Zhang, Z.; Jin, Y.; Niu, Y.; Cao, H.; Liang, X.; Chen, L.; Wang, J.; Peng, X. Solution-Processed, HighPerformance Light-Emitting Diodes Based on Quantum Dots. Nature 2014, 515, 96-99.

(22) Li, J.; Shan, X.; Bade, S. G. R.; Geske, T.; Jiang, Q.; Yang, X.; Yu, Z. Single-Layer Halide Perovskite Light-Emitting Diodes with Sub-Band Gap Turn-On Voltage and High Brightness. J. Phys. Chem. Lett. 2016, 7, 4059-4066.

(23) Schubert, E. F. Light-Emitting Diodes, 2nd ed.; Cambridge University Press: Cambridge, 2006.

(24) Greenham, N. C.; Friend, R. H.; Bradley, D. D. C. Angular Dependence of the Emission from a Conjugated Polymer Light-Emitting Diode: Implications for Efficiency Calculations. Adv. Mater. 1994, 6, 491-494.

(25) Di Stasio, F.; Christodoulou, S.; Huo, N.; Konstantatos, G. Near-Unity Photoluminescence Quantum Yield in $\mathrm{CsPbBr} 3$ Nanocrystal Solid-State Films via Post-Synthesis Treatment with Lead Bromide. Chem. Mater. 2017, 29, 7663-7667. 
(26) Chin, X. Y.; Perumal, A.; Bruno, A.; Yantara, N.; Veldhuis, S. A.; Martínez-Sarti, L.; Chandran, B.; Chirvony, V.; Lo, A. S.-Z.; So, J.; Soci, C.; Grätzel, M.; Bolink, H. J.; Mathews, N.; Mhaisalkar, S. G. Self-Assembled Hierarchical Nanostructured Perovskites Enable Highly Efficient LEDs via an Energy Cascade. Energy Environ. Sci. 2018, 11, 1770-1778.

(27) Zhang, X.; Wang, W.; Xu, B.; Liu, H.; Shi, H.; Dai, H.; Zhang, X.; Chen, S.; Wang, K.; Sun, X. W. Less-Lead Control toward Highly Efficient Formamidinium-Based Perovskite Light-Emitting Diodes. ACS Appl. Mater. Interfaces 2018, 10, 24242-24248.

(28) Yang, X.; Zhang, X.; Deng, J.; Chu, Z.; Jiang, Q.; Meng, J.; Wang, P.; Zhang, L.; Yin, Z.; You, J. Efficient Green Light-Emitting Diodes Based on Quasi-Two-Dimensional Composition and Phase Engineered Perovskite with Surface Passivation. Nat. Commun. 2018, 9, 570.

(29) Lorenzon, M.; Sortino, L.; Akkerman, Q.; Accornero, S.; Pedrini, J.; Prato, M.; Pinchetti, V.; Meinardi, F.; Manna, L.; Brovelli, S. Role of Nonradiative Defects and Environmental Oxygen on Exciton Recombination Processes in CsPbBr ${ }_{3}$ Perovskite Nanocrystals. Nano Lett. 2017, 17, 3844-3853.

(30) Meggiolaro, D.; Mosconi, E.; De Angelis, F. Mechanism of Reversible Trap Passivation by Molecular Oxygen in Lead-Halide Perovskites. ACS Energy Lett. 2017, 2, 2794-2798.

(31) Kumar, S.; Jagielski, J.; Kallikounis, N.; Kim, Y.-H.; Wolf, C.; Jenny, F.; Tian, T.; Hofer, C. J.; Chiu, Y.-C.; Stark, W. J.; Lee, T.-W.; Shih, C.-J. Ultrapure Green Light-Emitting Diodes Using Two-Dimensional Formamidinium Perovskites: Achieving Recommendation 2020 Color Coordinates. Nano Lett. 2017, 17, 5277-5284.

(32) Giovanella, U.; Pasini, M.; Lorenzon, M.; Galeotti, F.; Lucchi, C.; Meinardi, F.; Luzzati, S.; Dubertret, B.; Brovelli, S. Efficient Solution-Processed Nanoplatelet-Based Light-Emitting Diodes with High Operational Stability in Air. Nano Lett. 2018, 18, 3441-3448.

(33) Di, D.; Romanov, A. S.; Yang, L.; Richter, J. M.; Rivett, J. P. H.; Jones, S.; Thomas, T. H.; Abdi Jalebi, M.; Friend, R. H.; Linnolahti, M.; Bochmann, M.; Credgington, D. High-Performance Light-Emitting Diodes Based on Carbene-Metal-Amides. Science 2017, 356, 159-163. 


\title{
Supporting information
}

\section{High-Efficiency Light-Emitting Diodes Based on Formamidinium Lead Bromide Nanocrystals and solution processed transport layers}

\author{
Francesco Di Stasio ${ }^{\dagger}$, Iñigo Ramiro ${ }^{\dagger}, \mathrm{Yu} \mathrm{Bi}^{\dagger}$, Sotirios Christodoulou ${ }^{\dagger}$, Alexandros Stavrinadis ${ }^{\dagger}$ and Gerasimos \\ Konstantatos ${ }^{\dagger, *}$ \\ +ICFO-Institut de Ciencies Fotoniques, The Barcelona Institute of Science and Technology, 08860 Castelldefels (Barcelona), Spain \\ *ICREA-Institució Catalana de Recerca i Estudis Avançats, Passeig Lluís Companys 23, 08010
}

\section{Chemicals:}

Lead(II) bromide ( $\mathrm{PbBr}_{2}, 99.999 \%$ trace metals basis), Formamidine acetate (FAAc, 99\%), Octylamine (OcAm, 99\%), Butylamine (ButAm, 99.5\%) Octanoic acid (OcAc, 98\%), Propionic acid (PrAc, > 99.5\%), Oleic acid (99\%), anhydrous Toluene (TOL, 99.8\%), Methyl acetate (>98\%), anhydrous Chlorobenzene (99.8\%) and anhydrous 1,4 dioxane (99.8\%) were purchased from Sigma-Aldrich. 1-propanol (PrOH, Pharmpur $\left.{ }^{\odot}\right)$ and n-Hexane (Hex, 99\%) were purchased from Sharlab. All chemicals were used without any further purification.

\section{Experimental:}

Perovskite nanocrystals synthesis and purification: The NCs were synthesized using the previously published procedure in ref. 25 of the main text with a modification: instead of using Cs-acetate dissolved in 1-propanol, we have employed a 1-propanol formamidine acetate solution $(18 \mathrm{mg} / \mathrm{ml})$. The as-synthesized NCs were then centrifuged at $1500 \mathrm{rpm}$ for 2 minutes and re-dispersed in $20 \mathrm{ml}$ of anhydrous toluene. A post-synthesis treatment with $\mathrm{PbBr}_{2}(185 \mathrm{mg} / \mathrm{ml})$ dissolved in 1:1:1 Propionic Acid:Butylamine:Hexane in volume was carried out to enhance the PLQY (see ref. $25,12 \mu$ for a typical synthesis). After the treatment, oleic acid (35 $\mu$ l for a typical synthesis) was added to the solution to dissolve the NC aggregates observed under TEM. The solution was then centrifuged at 1000 $\mathrm{rpm}$, the supernatant was collected and transferred into a new vial. An equal volume of anhydrous Methyl acetate ( $20 \mathrm{ml}$ in a typical reaction) was added to the solution and centrifuged once more at $2500 \mathrm{rpm}$ for 5 minutes, the supernatant was discarded and the precipitate re-dissolved in $500 \mu \mathrm{l}$ anhydrous toluene. Finally, the obtained NC solution was ready for film preparation. The entire procedure was carried out in air and at room temperature.

Transmission Electron Microscope: Imaging was carried out with a JEOL JEM-2100 LaB6 transmission electron microscope, operating at $200 \mathrm{kV}$. The spectrometer is an Oxford Instruments INCA x-sight, with a Si(Li) detector. Samples for TEM characterization were prepared by drop-casting diluted NC solutions onto 300-mesh carbon-coated copper grids.

Ultraviolet photoelectron spectroscopy: UPS measurements were performed with a SPECS PHOIBOS 150 hemispherical analyzer (SPECS GmbH, Berlin, Germany) in ultra-high vacuum conditions ( $\left.10^{-10} \mathrm{mbar}\right)$ using a monochromatic HeI UV source $(21.2 \mathrm{eV})$ as excitation. 
Device cross section: The cross sectional images of the device were obtained using a Zeiss Augira cross-beam workstation. A layer of platinum (Pt, around $200 \mathrm{~nm}$ ) was deposited via gas injection system while the cross-section cut was made with a gallium focus ion beam (Ga-FIB). The SEM image was obtained using a voltage of $5 \mathrm{kV}$ and aperture size of $30 \mu \mathrm{m}$ with an Inlens detector.

Optical characterization: Optical absorption spectra were collected using a Varian Cary-5000 UV-Vis-NIR spectrophotometer. Photoluminescence (PL) measurements were performed using a Horiba Jobin Yvon iHR550 Fluorolog system coupled to a Horiba TBX-04 photomultiplier tube, a calibrated Quanta-phi integrating sphere and a FluoroHub time-correlated single photon counting card. All steady-state PL spectra were corrected for the system response function and were collected using a Xenon-lamp coupled with a monocromator as excitation source. For time-resolved PL measurements a pulsed laser diode was employed (Horiba Nanoled, $\lambda=405 \mathrm{~nm}$, pulse full-width-half-maximum of $50 \mathrm{ps}$, fluence $\approx 1 \mathrm{~nJ} / \mathrm{cm}^{2}$ ). All photoluminescence quantum yield (PLQY) measurements were carried out in the integrating sphere $\left(\lambda_{\text {exc }}=380 \mathrm{~nm}, \Delta \lambda=5 \mathrm{~nm}\right.$, power density $\left.=1 \mathrm{~mW} / \mathrm{cm}^{2}\right)$. FAPbBr $\mathrm{NCs}_{3}$ solutions for PLQY were prepared in quartz cuvettes and diluted to 0.1 optical density at the excitation wavelength and the measurements were carried out in a $4 \pi$ configuration. For PLQY on films, the measurements were carried out in a $2 \pi$ configuration, and all films were prepared via spincoating at $2000 \mathrm{rpm}$ on soda-lime glass substrates $\left(\right.$ area of $\left.1 \mathrm{~cm}^{2}\right)$.

Device fabrication: Glass slides with patterned ITO were used as substrates and they were cleaned initially with soapy water, rinsed then immersed in acetone for 30 minutes, and finally rinsed with isopropanol. The electron transport layer was deposited via spincoating in air at $3000 \mathrm{rpm}$ for $30 \mathrm{sec}$ of a $\mathrm{ZnO}$ nanoparticle $(40 \mathrm{mg} / \mathrm{ml})$ chloroform solution ( $\mathrm{ZnO}$ nanoparticles were previously synthesized using the procedure in ref. 19 of the main text). The spin-coating step was repeated a second time to obtain the desired thickness $(80 \mathrm{~nm})$ followed by annealing in air at $260 \mathrm{C}$ for 30 minutes. The $\mathrm{FAPbBr}_{3} \mathrm{NC}$ layer was spin-coated in air at $2000 \mathrm{rpm}$ for 20 sec (solution concentration $\approx 120 \mathrm{mg} / \mathrm{ml}$, thickness $=95 \mathrm{~nm}$ ); the film RMS was measured with a Dektak Profilometer. Afterward, the glass/ITO/ZnO/FAPbBr 3 NC layer substrates were transferred in an ALD system (GEMStar XT Thermal, Arradiance inc.) inside a Nitrogen filled glove-box where they were exposed to 3 pulses of trimethylaluminium to carry out the ligand cross-linking step. The substrates were then left in air for $24 \mathrm{~h}$ and after transferred into a nitrogen filled glove-box where the hole-transport layer was spincoated using a $10 \mathrm{mg} / \mathrm{ml}$ poly vinyl carbazole (PVK) solution in 1,4-dioxane and a $10 \mathrm{mg} / \mathrm{ml}$ Poly[N,N'-bis(4-butylphenyl)-N,N'bisphenylbenzidine] (PTPD) in chlorobenzene; both polymers were spincoated at $2000 \mathrm{rpm}$ for $30 \mathrm{sec}$ (total thickness: $95 \mathrm{~nm}$ ). The $50 \mathrm{~nm}$ Platinum electrode was deposited using a shadow mask and a RC magnetron sputtering system (AJA Orion 8 HV) at room temperature.

Device characterization: The current-voltage-luminance characteristics were measured using a Keithley 2636A source-measure unit coupled to a calibrated PDA 100A Si switchable gain detector from Thorlabs. The system was controlled via a LabView interface. The output of the Si detector was converted into power (photon flux) using the responsivity of the detector. The EQE was calculated as the ratio of the photon flux and the driving current of the device. The EL spectra of the devices were collected using the Horiba Jobin Yvon iHR550 Fluorolog system previously described while the bias was manually applied with a Keithley 2636A source-measure unit. 


\section{Ultraviolet Photoelectron spectroscopy:}
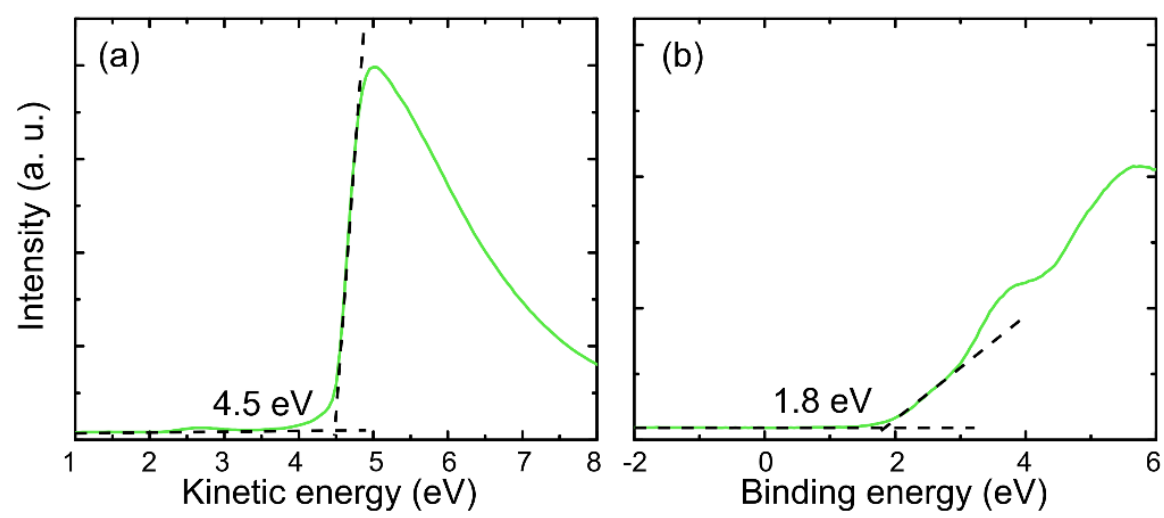

Figure S1 UPS spectrum of a $\mathrm{FAPbBr}_{3} \mathrm{NC}$ film prepared via spin-coating on ITO glass. (a) Secondary electron emission as a function of the kinetic energy of the photoelectrons. From the onset of the photoemission a work function of $(4.5 \pm 0.2)$ eV can be estimated for the $\mathrm{FAPbBr}_{3} \mathrm{NC}$ film. (b) Valence band maximum (VBM) relative to the Fermi level. From the VBM onset a value of $1.8 \pm 0.2 \mathrm{eV}$ is extracted, thus obtaining a position of the VBM with respect to the vacuum level of $-6.3 \pm 0.2 \mathrm{eV}$.

\section{Current density-luminance-voltage curves of the "champion" device in logarithmic scale:}

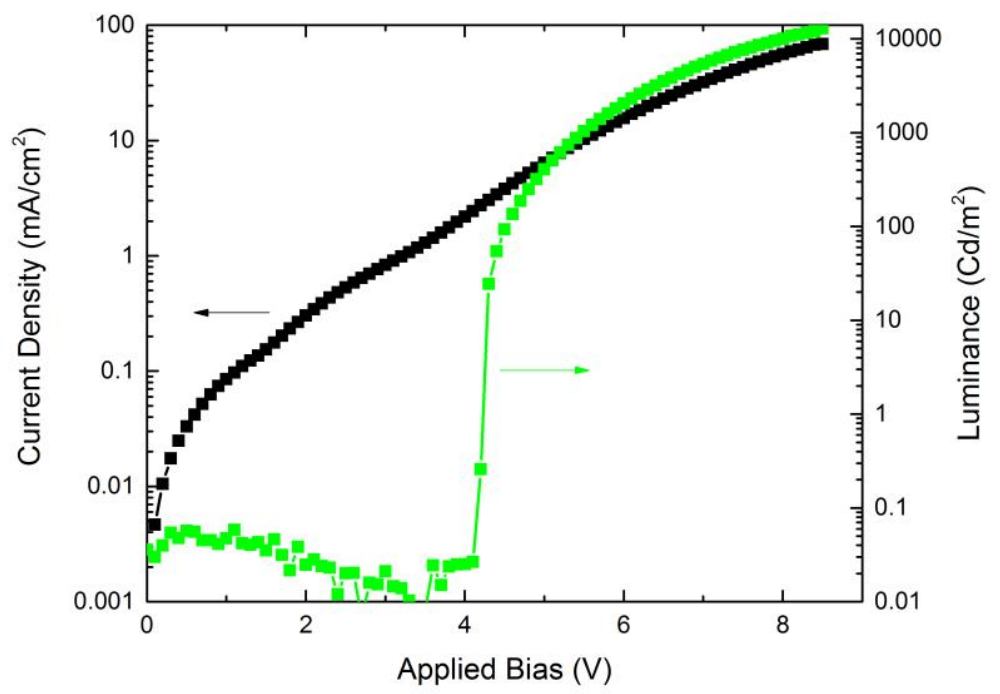

Figure S2 JVL curves of the "champion" device plotted in logarithmic scale for clarity. 
CIE 1931 colour coordinates values:

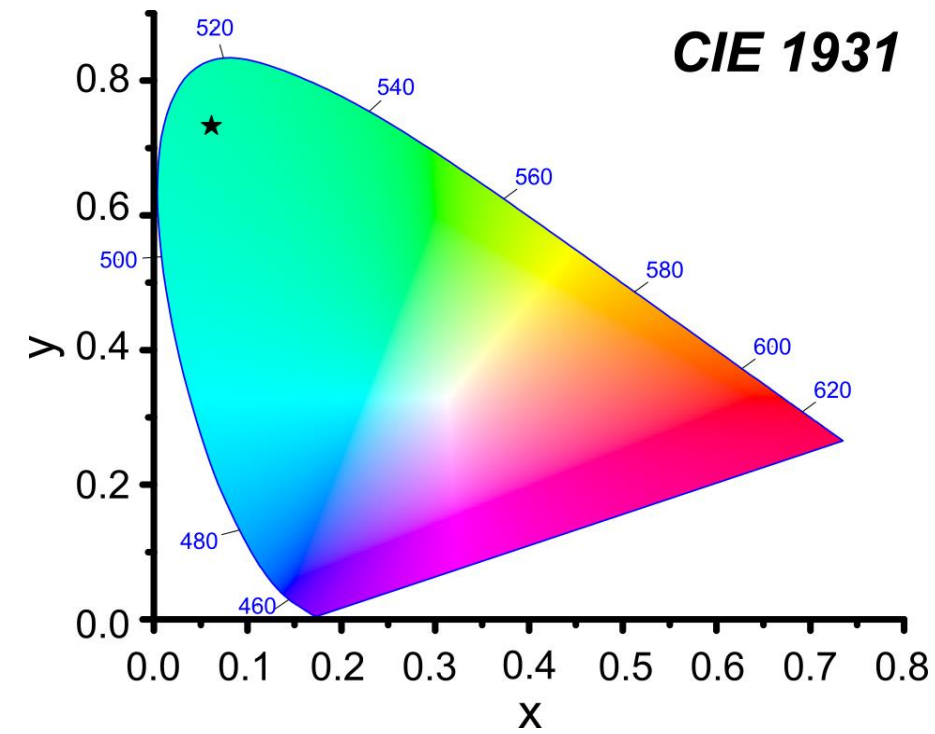

Figure S3 CIE 1931 diagram for $\mathrm{FAPbBr}_{3} \mathrm{NC}$ LEDs. The obtained color coordinates are $\mathrm{x}=0.0613$ and $\mathrm{y}=0.732$ 


\section{LED performance VS FAPbBr ${ }_{3} \mathrm{NC}$ film thickness:}

Table $\mathrm{S} 1$ key LED performance parameters variation upon $\mathrm{FAPbBr}_{3} \mathrm{NC}$ film thickness increase

\begin{tabular}{ccc}
\hline Thickness $(\mathrm{nm})$ & $\mathrm{VON}(\mathrm{V})$ & $\operatorname{EQE}_{\mathrm{MAx}}(\%)$ \\
\hline 120 & 4.7 & 5.31 \\
95 & 4.1 & 6.04 \\
60 & 3.2 & 3.72 \\
40 & 2.9 & 2.45
\end{tabular}

\section{JVL curve acquired after first scan:}

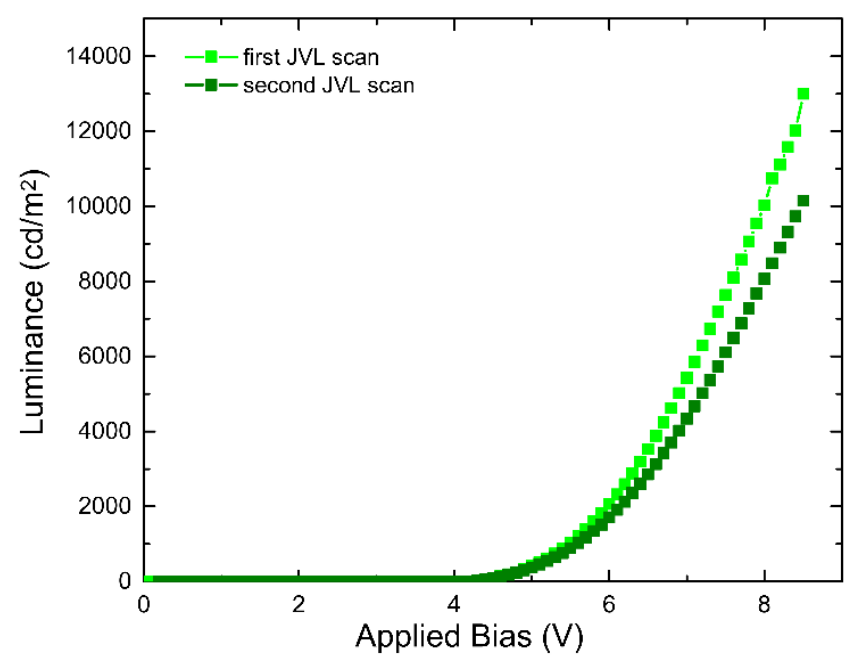

Figure S4 Device stability remains very limited despite the good overall performance of the LEDs. A JVL scan carried out directly after the intial one already present a decrease in Luminance from $12998 \mathrm{~cd} / \mathrm{m}^{2}$ to $10147 \mathrm{~cd} / \mathrm{m}^{2}$ 
TEM micrograph of the $\mathrm{FAPbBr}_{3} \mathrm{NCs}$ before and after purification:
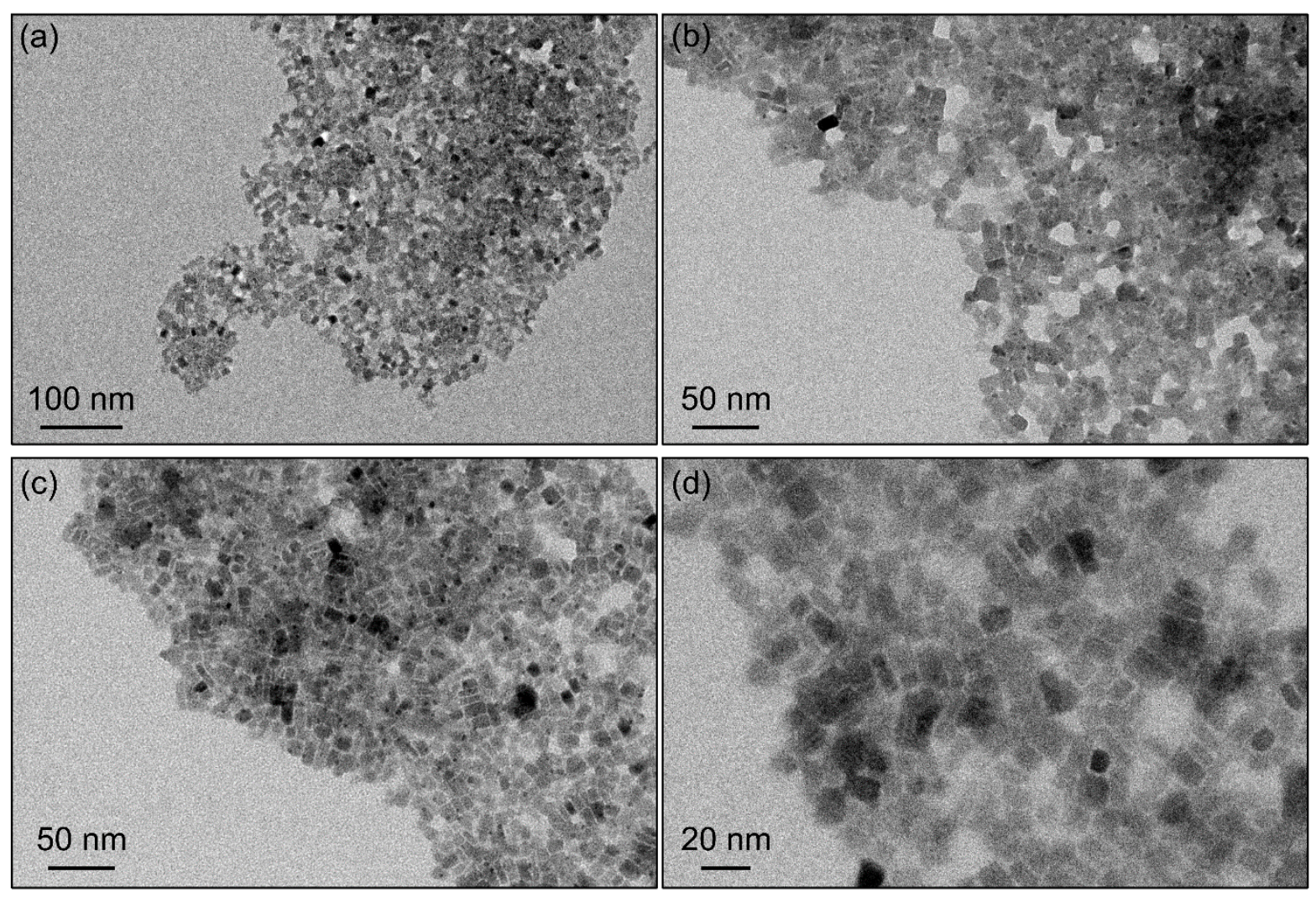

Figure S5 TEM micrographs at different magnifications of the $\operatorname{FAPbBr}_{3} \mathrm{NCs}$ before $(\mathrm{a}, \mathrm{b})$ and after $(c, d)$ the addition of oleic acid during purification. The as-synthesized NCs appear aggregated before addition of the oleic acid, as observed in similar synthetic methods emplyoing short ligands and carried out at room temperature. 


\section{Effect of the addition of oleic acid to the $\mathrm{FAPbBr}_{3} \mathrm{NC}$ solution:}

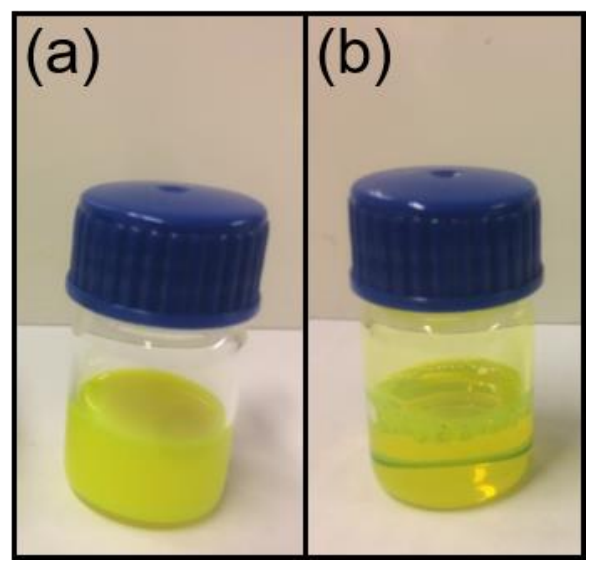

Figure S6 Photo of the $\mathrm{FAPbBr}_{3} \mathrm{NC}$ solution before (a) and after (b) the oleic acid addition. The NC solution turns from turbid to clear.

\section{Total volumes used in a typical reaction:}

Table $S 2$ Total solvent volumes and precursors weigths used in a typical reaction of FAPbBr 3 NCs. $6 \mathrm{ml}$ of the FA precursor solution (18 mg/ml) was first added to the Hexane and 1-propanol mixture. Afterward, $7 \mathrm{ml}$ of the $\mathrm{PbBr}_{2}$ precursor solution (185 mg/ml) was quickly injected into the flask. (see reference 25 of the main text)

\begin{tabular}{lccc}
\hline Chemicals & Reaction flask & $\begin{array}{c}\text { Formamidinium precursor } \\
\text { solution }\end{array}$ & $\begin{array}{c}\mathrm{PbBr}_{2} \text { precursor } \\
\text { solution }\end{array}$ \\
\hline Hexane & $48 \mathrm{ml}$ & $/$ & $/$ \\
1-propanol & $18 \mathrm{ml}$ & $6 \mathrm{ml}$ & $2.33 \mathrm{ml}$ \\
Octanoic acid & $/$ & $/$ & $2.33 \mathrm{ml}$ \\
Octylamine & $/$ & $/$ & $2.33 \mathrm{ml}$ \\
Formamidine acetate & $/$ & $108 \mathrm{mg}$ & $/$ \\
$\mathrm{PbBr}_{2}$ & $/$ & $/$ & $1295 \mathrm{mg}$ \\
\hline
\end{tabular}

\section{PL decays fitting parameters:}

Table S3 PL decays were fitted with a two-exponential function: $I=I_{0}+I_{1} e^{\left(-t_{y} / T_{1}\right)}+I_{2} e^{\left(-t / T_{2}\right)}$ using the following parameters

\begin{tabular}{lcccc}
\hline Sample & $\mathrm{I}_{1}$ & $\tau_{1}(\mathrm{~ns})$ & $\mathrm{I}_{2}$ & $\tau_{2}(\mathrm{~ns})$ \\
\hline $\begin{array}{l}\text { FAPbBr } \\
\text { purification }\end{array}$ & 0.71 & 2.66 & 0.29 & 14.1 \\
$\begin{array}{l}\text { FAPbBr } \\
\text { cation }\end{array}$ & 0.75 & 4.27 & 0.25 & 13.85 \\
\hline
\end{tabular}




\section{Cross-linking of the $\mathrm{FAPbBr}_{3} \mathrm{NC}$ film with TMA vapours:}

The cross-linking of the film was performed in a GEMStar XT Thermal ALD system. High-purity TMA was purchased from STREM Chemicals Inc. The process was carried out close to ambient temperature $\left(29-33^{\circ} \mathrm{C}\right)$. Before the process, the reaction chamber was pumped down and subsequently filled with pure nitrogen up to a pressure of typically 1.3-1.4 mbar. The TMA manifold was maintained at $150^{\circ} \mathrm{C}$ during gas supply. TMA was applied in 9-ms pulses (longer pulses were found to quench the film luminescence) at a partial pressure of 0.04 mbar. The dosage of TMA in different samples depends on the number of TMA pulses applied.
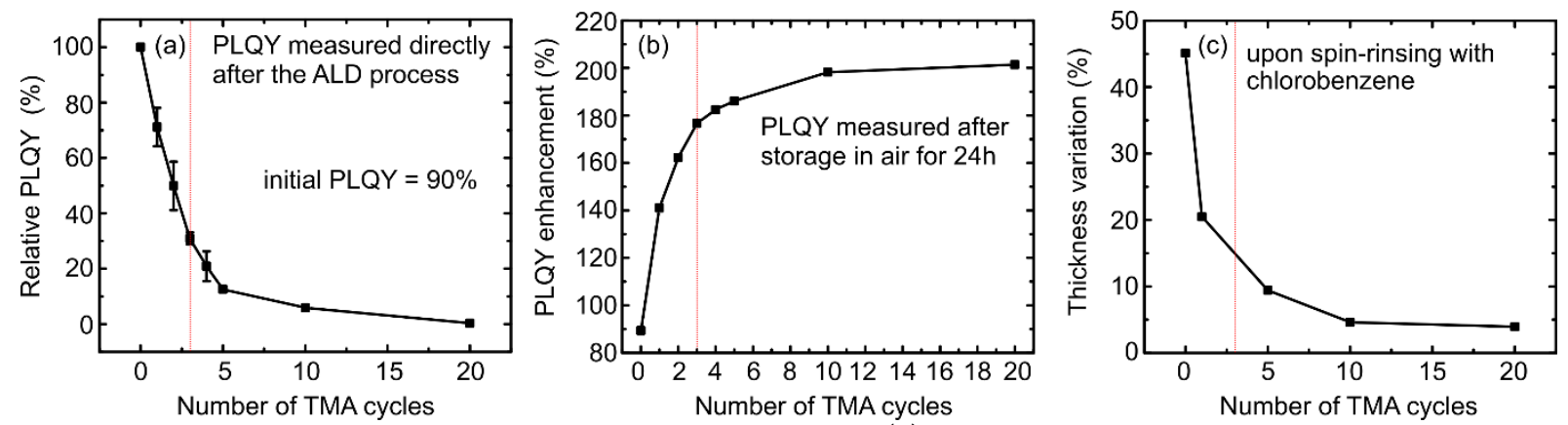

Figure S7 Effect of the TMA vapours treatment on the FAPbBr 3 NC film. (a) PLQY variation after the treatment vs number of TMA vapours cycles. (b) PLQY recovery and enhancement after exposure to air for $24 \mathrm{~h}$ vs number of TMA vapours cycles previously applied. (c) NC Film thickness reduction upon spin-rinsing with chlorobenzene vs number of $\mathrm{TMA}$ vapours cycles. $\mathrm{The} \mathrm{FAPbBr}_{3} \mathrm{NC}$ film used in the LED was exposed to 3 pulses of TMA (highlighted with dotted red line in all panels) thus obtaining a film with a retention rate of $85 \%$ and a final PLQY of $46 \%$.
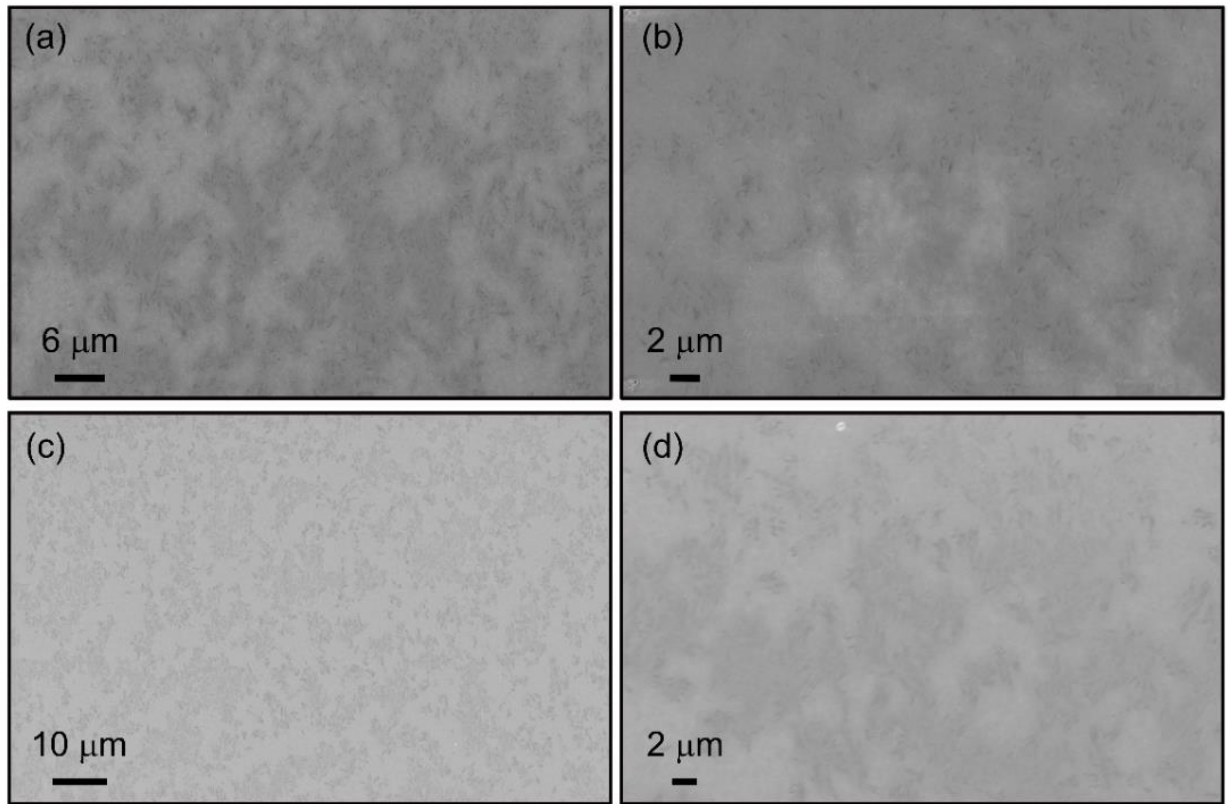

Figure S8 SEM images of the $\mathrm{FAPbBr}_{3} \mathrm{NC}$ film before $(\mathrm{a}, \mathrm{b})$ and after $(\mathrm{c}, \mathrm{d})$ exposure to TMA vapours. No cracks or sizeable changes in film morphology are observed. 